\title{
Gas-Phase Proton Transfer Reactions Involving Multiply Charged Cytochrome $c$ Ions and Water Under Thermal Conditions
}

\author{
Brian E. Winger, Karen J. Light-Wahl, and Richard D. Smith \\ Chemical Methods and Separations Group, Chemical Sciences Department, Pacific Northwest Laboratory, \\ Richland, Washington, USA
}

\begin{abstract}
Investigations of gas-phase proton transfer reactions have been performed on protein molecular ions generated by electrospray ionization (ESI). Their reactions were studied in a heated capillary inlet/reactor prior to expansion into a quadrupole mass spectrometer. Results from investigations involving protonated horse heart cytochrome $c$ and $\mathrm{H}_{2} \mathrm{O}$ suggest that Coulombic effects can lower reaction barriers as well as aid in entropically driven reactions. For example, the charge state distribution observed by a quadrupole mass spectrometer for multiply protonated cytochrome $c$ without the addition of any reactive gas ranges from $9+$ to $19+$, with the $[\mathrm{M}+15 \mathrm{H}]^{15}+$ ion being the most intense peak. With the addition of $\mathrm{H}_{2} \mathrm{O}$ (proton affinity approximately $170.3 \pm 2 \mathrm{kcal} / \mathrm{mol}$ ) to the capillary reactor at $120{ }^{\circ} \mathrm{C}$, the charge state distribution shifts to a lower charge, ranging from $13+$ to less than $9+$. Under the same conditions with argon (proton affinity approximately $100 \mathrm{kcal} / \mathrm{mol}$ ) as the reactive gas, no shift in the charge state distribution is observed. The results demonstrate that proton transfer to water can occur for highly protonated molecular ions, a process that would be expected to be highly endothermic for singly protonated molecules (for which Coulombic destabilization is not significant). The results imply that the charge state distribution from ESI is somewhat dependent upon the mechanism and speed of the droplet evaporation/ion desolvation process, which may vary substantially with the ESI/mass spectrometry interface design. (I Am Soc Mass Spectrom 1992, 3, 624-630)
\end{abstract}

$\mathrm{T}$ The recent advent of soft ionization methods based on electrospray ionization (ESI) [1-4], arguably the softest of all ionization methods, is rapidly transforming mass spectrometry into an extremely powerful analytical technique for biomolecule analysis. A key feature of ESI is the formation of multiply charged ions for macromolecules by a process presumably related to their charge state in solution (or, more appropriately, the charged droplet). To date the majority of macromolecular species investigated using ESI have been sufficiently charged that even compounds whose average molecular weight are up to $5 \mathrm{MDa}$ (megadalton) can be detected [5].

The formation of gas-phase multiply charged macromolecules offers a unique opportunity for investigating the gas-phase structure and reactivity of such species using mass spectrometric techniques. Recently, McLuckey et al. [6] have shown that multiply charged cytochrome $c$ can undergo a proton transfer reaction with dimethylamine, producing higher mass-to-charge

Address reprint requests to Richard D. Smith, Chemical Methods and Separations Group, Chemical Sciences Department, P8-19, Pacific Northwest Laboratory, Richland, WA 99352. ratio species. These reactions are presumed to proceed via proton transfer from the highly protonated macromolecule, resulting in the formation of protonated amine and a lower charge state macromolecule. Subsequently, with the use of 1,6-diaminohexane, McLuckey et al. have shown the feasibility of determining the charge state of product ions from tandem mass spectrometry studies (MS/MS) (for molecular ions) and MS/MS/MS (for fragment ions), in which an ion-molecule reaction leading to proton transfer is performed prior to the final stage of mass analysis [7]. The fact that these reactions occur might initially be surprising, because the proton affinities normally attributed to the basic amino acid residues (greater than $230 \mathrm{kcal} / \mathrm{mol}$ ) are generally higher than that of the amines [8]. However, by invoking the destabilizing effect of Coulombic repulsion such reactions can be rationalized.

More recently, a simple Y-tube apparatus has been developed by Ogarzalek Loo et al. [9] for the purpose of investigating near-thermal energy ion-ion reactions and, subsequently, ion-molecule reactions [10] of multiply charged molecules near atmospheric pressure and prior to expansion into vacuum. These data indicate that complex interactions between the macromolecular 
species and the reagent ion or molecule can occur in the capillary reaction region. Specifically, the ion-molecule reaction studies indicate an acute tendency of the original ion population to undergo proton transfer reactions with various amines, resulting in the shift of the original charge state distribution to higher mass-to-charge ratio (i.e., lower charge state) and, in some cases, beyond the $m / z 2000$ limit of the mass spectrometer [10].

The capability of performing ion-molecule reactions prior to expansion and mass analysis has led us to investigate reactions of multiply charged proteins in an attempt to qualitatively probe the gas-phase structure of these large ions. This article presents the results of the reaction of protonated horse heart cytochrome $c$ with water and argon. These investigations have shown that proton transfer reactions between the multiply protonated protein and water can occur, even though the proton affinity of water is much less than that of the individual basic amino acid residues. This observation also concurs with results reported by McLuckey et al. $[6,7]$ and Ogorzalek Loo et al. [10], as well as with a preliminary report by Chait et al. [11], in which they suggested that the observed ESI distribution reflects the ionization of proteins in solution, the charge state distribution is likely modified by subsequent deprotonation reactions in the gas phase and by the projected Coulombic contributions to each of these reactions [11]. The present results illustrate the large role of Coulombic energy in the ion-molecule chemistry of multiply charged macromolecules and have implications for the design of ESI interfaces.

\section{Experimental}

The electrospray liquid sheath assembly [12] and the modified Sciex (Thornhill, Ontario, Canada) TAGA $6000 \mathrm{E}$ triple quadrupole mass spectrometer [13] used for these experiments are described elsewhere. To facilitate the study of reactions prior to expansion, the capillary inlet/reactor assembly depicted in Figure 1 replaces the interface typically used. This assembly consists of a 10-cm-long stainless steel inlet capillary, 0.020 in. i.d. $\times 1 / 16$ in. o.d. (A), a standard stainless steel $1 / 16$ in. Swagelok mixing tee (B), and a $20-\mathrm{cm}-$ long stainless steel reaction capillary, 0.020 in. i.d. $\times$ $1 / 16$ in. o.d. (C), which is attached to a flange consisting of a $1 / 8$ in. Swagelok fitting. A $1 / 16$ in. i.d. $\times 1 / 8$ in. o.d. graphite ferrule (Alltech Associates Inc., Deerfield, ILJ is used to form the vacuum seal about the

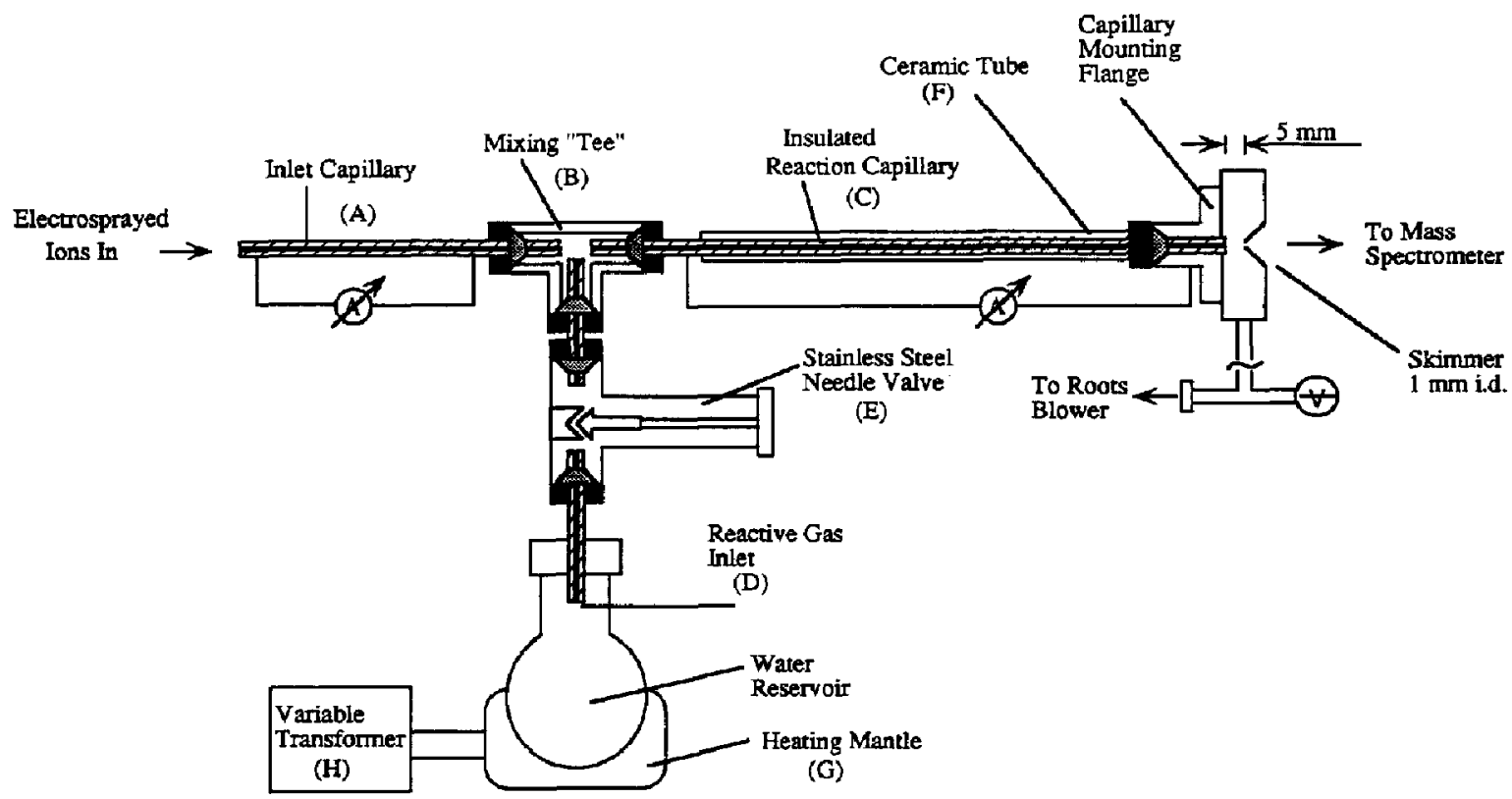

Figure 1. Capillary interface/reactor used to perform ion-molecule reactions prior to expansion into vacuum. The assembly consists of a 0.020-in. i.d $\times 1 / 16-i n$. o.d., 10-cm-long inlet capillary (A), a standard 1/16-in. stainless steel Swagelok tee (B), and a 0.020-in. i.d. $\times 1 / 16$-in. o.d., 20-cm-long reaction capillary (C). The reagent gas is added to the assembly at (D), while a stainless steel needle valve $(E)$ is used to isolate the reaction inlet from the ion inlet system. Normally, a $500-\mathrm{mL}$ round bottom flask containing doubly distilled and deionized water is attached to the reactive gas inlet. A heating mantle $(G)$ and a variable transformer $(H)$ are used to regulate water temperature. These are removed to add argon gas. An insulating ceramic tube (F) surrounds the reaction capillary to maintain a consistent temperature, while fiberglass insulation is wrapped around the mounting flange. 
exterior of the reaction capillary. To minimize temperature variations in the reaction capillary, as well as near the expansion end of the capillary, the reaction capillary is surrounded by a $17-\mathrm{cm}$-long, $0.093 \mathrm{in}$. i.d. $\times 0.250$ in. o.d. insulating ceramic tube $(F)$, and the mounting flange is wrapped with fiberglass insulation. The inlet and reaction capillaries are independently heated resistively, using two Electronic Measurement Inc. (Neptune, NJ) model 20550 1000-W TCR high-current power supplies. The external temperature of each capillary is monitored using a chromel-alumel (type K) thermocouple and an Omega Engineering, Inc. (Stanford, CT) CN-9000 thermocouple monitor. Typical temperatures for the inlet and reaction capillaries are $120^{\circ} \mathrm{C}$ for the spectra reported, and similar results have been obtained between 90 and $150^{\circ} \mathrm{C}$. Both capillaries are typically biased to $+130 \mathrm{~V}$, while the sampling skimmer is biased to $+65 \mathrm{~V}$. For experiments that involve the comparison of dissociation (of the molecular ions) and desolvation (of the ions which have been allowed to react with $\mathrm{H}_{2} \mathrm{O}$ ), the capillary voltage is increased to increase the capillary-skimmer gradient, $\Delta C S$. This provides an independent mechanism to examine the effect of additional collisional heating in the capillary-skimmer region after expansion, and (if desired) to remove residual solvation.

The reaction gas is added to the analyte through a third transfer capillary connected to the third arm of the tee (D). The entire transfer line and Swagelok tee are wrapped with heating tape and heated to $100{ }^{\circ} \mathrm{C}$ to prevent condensation of water vapor. A stainless steel needle valve (E) is located between the tee and the reactive gas inlet to allow for isolation from the ion inlet system. For experiments with water, a $50-\mathrm{mL}$ aliquot of doubly distilled and deionized water is added to a 500-mL round bottom flask and heated to approximately $100{ }^{\circ} \mathrm{C}$ using a heating mantle $(G)$ and a variable transformer $(\mathrm{H})$. A chromel-alumel (type $\mathrm{K}$ ) thermocouple (connected to an Omega 1602-K thermocouple monitor) is placed above the water level to monitor the vapor temperature. In this manner a stable and reproducible flow of water vapor could be introduced into the reaction region. For studies with argon, gas is added to the reaction vessel at point (D), the inlet to the reaction gas transfer line. This allows for argon to be heated to approximately the same temperature as the water prior to entering the reaction region (see Figure 1). With the expansion end of the reaction capillary approximately $5 \mathrm{~mm}$ from the sampling skimmer, pressure in the capillary-skimmer region is typicalty 0.17 torr (at the guage location) when the capillaries are heated, as measured by a thermocouple downstream of the interface region. The measured pressure in this region increases to approximately 0.30 torr when water is added, and decreases to approximately 0.10 torr when Ar at $10 \mathrm{psi}$ is added. Although the actual pressure of each gas at the interface could not be directly determined, the difference in the measured values can most likely be attributed to sensitiv- ity differences of the thermocouple gauge for the different gases at these pressures [14]. The purity of the water, the reproducibility of the reported results over extended experimental studies, and the use of many different water samples provide confidence that the present results are not due to trace impurities in the water having substantially higher proton affinities.

The horse heart cytochrome $c$ used was obtained from Sigma Chemical Co. (St. Louis, Mo.) and used without purification. Solutions consisting of $0.5 \mu \mathrm{g} / \mu \mathrm{L}$ cytochrome $c$ in $47.5 / 47.5 / 5.0 \% \mathrm{v} / \mathrm{v} / \mathrm{v} \quad \mathrm{H}_{2} \mathrm{O}$ / methanol/acetic acid were used for the investigations.

\section{Results}

Figure 2a illustrates a typical ESI mass-to-charge ratio spectrum of horse heart cytochrome $\mathcal{c}_{r}$ in which the molecular ion charge state distribution ranges from $9+$ to $19+$ and the base peak corresponds to $[\mathrm{M}+$ $15 \mathrm{H}]^{15+}$. This spectrum was obtained with the heated capillary inlet/reactor. The calculated molecular

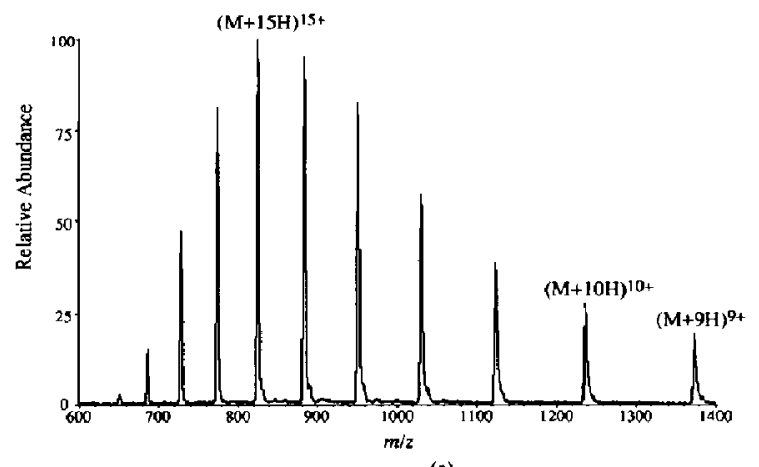

(a)

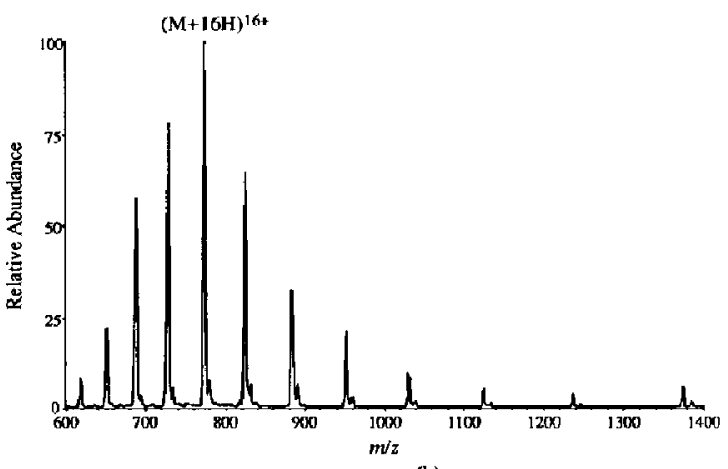

(b)

Figure 2. (a) Electrospray ionization (ESI) mass spectrum of horse heart cytochrome $c$ acquired using the capillary-skimmer interface illustrated in Figure 1. Both the inlet and reaction capillary temperatures were approximately $120^{\circ} \mathrm{C}$ and $\Delta C S$ was +65 V. (b) ESI mass spectrum of same sample as in (a) but acquired using the nozzle-skimmer interface and countercurrent nitrogen flow for droplet evaporation. 
weight of cytochrome $c$ from this distribution is 12,359 $\pm 1.5 \mathrm{Da}$, in good agreement with the actual molecular weight of $12,360 \mathrm{Da}$. Figure $2 \mathrm{~b}$ is a typical mass spectrum of cytochrome $c$ obtained with the more traditional nozzle-skimmer interface, which uses countercurrent gas $\left(\mathrm{N}_{2}\right)$ to cause droplet evaporation prior to the sampling orifice and expansion into vacuum. The mass spectrum illustrated in Figure 2a exhibits qualitatively less protonation than the spectrum in Figure $2 b$ which, on the basis of the results presented below, we attribute to the increased concentration of solvent species present in the capillary inlet system.

When water is added through the mixing tee (by heating the water flask to approximately $100{ }^{\circ} \mathrm{C}$ ) the charge state distribution shifts to lower charge state, resulting in molecular ions ranging from $9+$ to $13+$, with the $[\mathrm{M}+10 \mathrm{H}]^{10+}$ ion being the base peak, as shown in Figure 3. (Note that the actual $9+$ molecular ion concentration may actually be higher than the $10+$ ion because of the general discrimination against higher mass-to-charge ratio species for quadrupole instruments under normal tuning conditions.) The $m / z 1400$ limit of the mass spectrometer prevents the determination of the extent of charge state distribution shifting below the $9+$ charge state.

The absolute ion abundances measured for the $[\mathrm{M}$ $+9 \mathrm{H}]^{9+}$ and $[\mathrm{M}+10 \mathrm{H}]^{10+}$ ions prior to the addition of $\mathrm{H}_{2} \mathrm{O}$ are 49,800 and 78,000 counts/s, respectively. After the addition of $\mathrm{H}_{2} \mathrm{O}$, the ion abundances increase to 84,400 for the $[\mathrm{M}+9 \mathrm{H}]^{9+}$ ion and 126,400 to the $[\mathrm{M}+10 \mathrm{H}]^{10+}$ ion. Assuming that the total ion flux entering the mass spectrometer is not drastically altered due to the addition of $\mathrm{H}_{2} \mathrm{O}$ in the tee region, these results suggest that a substantial amount of ion signal lies beyond $m / z \quad 1400$, and hence at lower charge state. In fact, any addition of gas through the tee decreases the gas flow and ion current through the inlet capillary and results in an additional, and approximately equivalent, reduction in the abundances of all charge states (as observed upon the addition of argon, discussed below). Thus, the shift to lower charge states

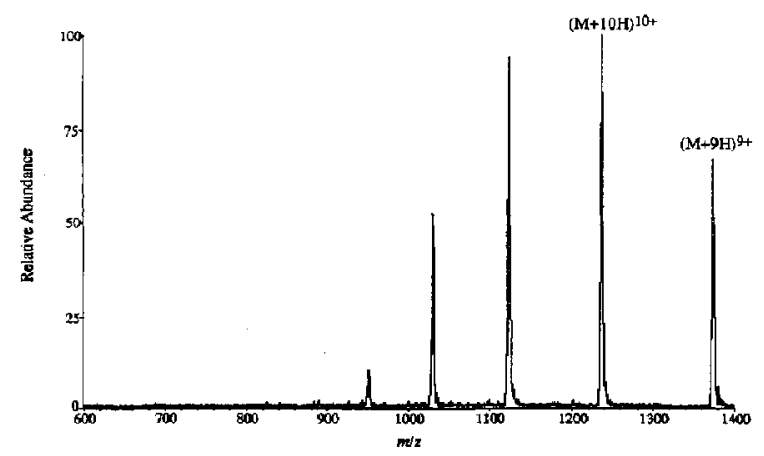

Figure 3. Electrospray ionization mass spectrum of horse heart cytochrome $c$ after water was added. The capillary temperatures were approximately $120^{\circ} \mathrm{C}$ and $\Delta C S$ was $+135 \mathrm{~V}$ (to reduce the increased solvation after addition of water). is likely somewhat greater than suggested by relative ion abundances before and after addition of water, and would best be studied by an instrument with an extended mass-to-charge ratio range.

Several parameters can affect the extent of proton transfer observed; however, the relative concentration of the reactive gas seems to be the most important. Varying the amount of water vapor added from 0.01 to 0.13 torr (pressure measured downstream from the interface region, not in the reaction capillary), the extent of proton transfer gradually increases until one obtains a mass spectrum such as the one shown in Figure 3. No molecular ions are observed in the massto-charge ratio range of the mass spectrometer beyond about 0.13 torr of added water (interface pressure).

As the amount of gas added increases, the amount of molecular ion solvation that occurs, either in the reaction capillary or because of condensation during expansion, also increases. To obtain the mass spectrum shown in Figure $2 \mathrm{a}$ (with no gas added), a $\triangle \mathrm{CS}$ of +65 $\mathrm{V}$ is used. However, the spectrum observed with this same $\triangle C S$ and 0.13 torr (interface pressure) of water vapor added shows molecular ion intensities reduced by about an order of magnitude, having extensive solvation indicated in the spectrum by a tail to higher mass-to-charge ratio. The mass spectrum illustrated in Figure 3 is obtained by increasing $\Delta C S$ to $+135 \mathrm{~V}$. This $\Delta C S$ increase results in improved intensity and symmetry of individual peaks via enhanced desolvation of the tail, but does not alter the observed charge distribution (i.e., the abundance at each molecular ion charge state is approximately the area of the unsolvated peak intensity and the solvated tail). An additional increase in $\Delta C S$ does not result in further desolvation of more highly charged species than are observed in Figure 3; rather, the collisionally activated dissociation of the existing molecular ions is observed [13]. To additionally verify the increased solvation with added water vapor, a mass spectrum was acquired with a $\triangle C S$ of $+135 \mathrm{~V}$, but with the valve between the $\mathrm{H}_{2} \mathrm{O}$ reservoir and the tee closed. Under these conditions the base peak is due to the heme group (at about $m / z$ 616.5), which is covalently bound to the polypeptide chain in cytochrome $c$. This observation can be attributed to dissociation of the more highly charged molecular ions [13]. This observation also suggests that ions in the reaction capillary may be somewhat more highly solvated prior to expansion. The greater solvation would reduce the likelihood of dissociation in the interface.

Under the conditions in which the mass spectrum in Figure 3 was obtained, $\mathrm{H}_{3} \mathrm{O}^{+}$was not observed. However, this can be rationalized on the basis of the reactive nature of the hydronium ion, the formation or possible participation of larger water clusters, and/or the interface's discrimination against low molecular weight components in the expansion. Therefore, to test further the hypothesized involvement of water in the observed proton transfer reactions, as opposed to other 
reactants, the experiments were repeated with Ar gas substituted for $\mathrm{H}_{2} \mathrm{O}$. The resulting mass spectrum shows no apparent charge state shifting (as compared to Figure 2a), even when more than $10 \mathrm{psi}$ of Ar (as measured on the tank regulator) was added to the mixing tee. Due to the method by which argon was added to the reaction region, increasing the pressure of argon increases the residence time of the analyte ions in the inlet capillary (before the tee), which may be useful for the study of kinetically slow reactions. Ar flow at the highest pressures served to greatly reduce, and ultimately eliminate, ESI signals, but no significant change in charge state distribution was observed. The greater opportunity for loss by interaction with the capillary wall apparently does not result in substantial discrimination based upon charge state. From thermochemical considerations one would not expect reaction with argon to be as efficient as $\mathrm{H}_{2} \mathrm{O}$ (proton affinity of Ar is about $100 \mathrm{kcal} / \mathrm{mol}[15]$ as compared to $170.3 \pm 2$ $\mathrm{kcal} / \mathrm{mol}$ for $\mathrm{H}_{2} \mathrm{O}$ [16]; no apparent reaction was observed. The assumption that $\mathrm{H}_{2} \mathrm{O}$ is the recipient of the proton transfer process appears reasonable. However, such an observation is initially surprising because the apparent proton affinities of the individual cytochrome $c$ charge sites are greater than 230 $\mathrm{kcal} / \mathrm{mol}$ on the basis of the values for individual amino acids. It should also be noted that similar deprotonation has been observed using other proteins, for example, myoglobin, bovine albumin, and ubiquitin, and with other reagent gases such as $\mathrm{D}_{2} \mathrm{O}$.

\section{Discussion}

The observed overall proton transfer reactions between $\mathrm{H}_{2} \mathrm{O}$ and multiply protonated cytochrome $c$ can be denoted by

$$
\begin{aligned}
(\mathrm{P}+ & n \mathrm{H})^{n+}+m \mathrm{H}_{2} \mathrm{O} \\
& \rightarrow(\mathrm{P}+(n-m) \mathrm{H})^{(n-m)+}+m \mathrm{H}_{3} \mathrm{O}^{+}
\end{aligned}
$$

where $\mathbf{P}$ denotes a protein molecule and $n$ and $m$ are integers, and can be explained by at least two different hypotheses. The apparent proton affinities of the individual amino acid residues in multiply protonated proteins probably differ from the corresponding singly charged amino acid residues because of intramolecular interactions and changes in the barriers to proton transfer reactions. It is also possible that the observed phenomenon is a result of an endothermic reaction that is entropically driven, that is, a positive entropy change that serves to make the reaction more exothermic [17]. We propose that both factors are relevant for such reactions and that both are related to Coulombic effects for multiply charged species.

Proton affinities of individual gas-phase amino acids have been experimentally determined using Fourier transform ion cyclotron resonance (ICR) [8], high-pres- sure mass spectrometry [18], and, more recently, laser desorption with ICR [19]. The determined values range from approximately $210 \mathrm{kcal} / \mathrm{mol}$ for glycine to more than $243 \mathrm{kcal} / \mathrm{mol}$ for arginine. Given an experimentally determined gas-phase proton affinity of 170.3 $\mathrm{kcal} / \mathrm{mol}$ for $\mathrm{H}_{2} \mathrm{O}$ [16], the proton transfer reaction between a protonated amino acid molecule and a water molecule is expected to be at least $40 \mathrm{kcal} / \mathrm{mol}$ endothermic and possibly more than $70 \mathrm{kcal} / \mathrm{mol}$ endothermic. However, the reactivity of an amino acid residue of a gas-phase multiply charged protein may vary markedly compared to expectations on the basis of proton affinities of the singly charged species. When a charged protein in solution is electrosprayed into the gas phase, the charge delocalization and screening by the relatively high dielectric constant of the solvent are substantially removed. This can result in destabilization of the multiply charged species via Coulombic repulsion of the nearly solvent-free charge sites. This, in turn, may induce the lowering of activation barriers for dissociative or other reaction processes [20]. Simplified theoretical calculations based on a "charge on a dissociating string" model support the barrier lowering postulated for dissociation along the backbone of a highly charged protein [20]. Similarly, it is reasonable that the apparent proton affinity values for various amino acid residues may decrease to an extent, depending upon the location of the amino acid residue relative to other charge sites. Such an explanation is also consistent with experimental results for reactions of multiply protonated ions with various amines $[6,7$, 10].

It should be noted that the use of the term "proton affinity" in the context of multiply charged molecules can be misleading. The proton affinity of a particular amino acid residue would correspond to the energy difference for reaction 2 :

$$
\mathrm{H}^{+}+(\mathrm{P}+m \mathrm{H})^{m+} \rightarrow(\mathrm{P}+(m+1) \mathrm{H})^{(m+1)+}
$$

By definition the proton affinity would include the energy needed to overcome the long-range repulsive Coulombic contribution. Since we are interested in proton transfer away from the multiply charged macromolecule, we are more generally concerned with only the much smaller short-range contribution relevant to a particular reaction coordinate. Most of the Coulombic energy is converted to relative translational energy of the products. Thus we might refer to a Coulombic effect as lowering the apparent proton affinity of a charge site, or define a new term (perhaps "intrinsic" or "short-range" proton affinity).

Unlike the barrier-lowering mechanism, there exist no reported results suggesting the possibility of an entropically driven reaction with respect to multiply charged species; however, a qualitative argument can be given for its possible occurrence. As discussed previously, Coulombic repulsions between the various 
charged sites increase with the elimination of solvent molecules because of the lowering or elimination of electrostatic screening. The resulting increase in Coulombic repulsion limits the possible range of structures for highly charged ions, therefore constraining their structure and torsional motion. Proton transfer would diminish the number of charge sites and decrease Coulombic forces, reducing such constraints. This resulting positive entropy change may be sufficiently large to facilitate reaction processes that would otherwise be endothermic.

The present results also suggest that design of interfaces to perform ionization via various techniques should take into account effects due to reactions with solvent molecules. In particular, it appears the resulting charge state distribution may be substantially dependent upon the manner in which evaporation/desolvation is conducted, and (likely) the rate of solvent removal. Indeed, qualitatively greater protonation is observed in our laboratory with the same mass spectrometer using a countercurrent gas flow for desolvation than for the heated capillary inlet interface (see Figure 2). In the latter case, desolvation occurs more slowly, and substantially higher solvent vapor concentrations will persist through the mass spectrometer interface. It is also a general observation that increasing the distance between the electrospray and the sampling orifice can lead to some discrimination against higher charge states. For example, in a recent study of the arginine tripeptide, it was noted that the $3+$ was attenuated more rapidly, apparently because of proton transfer to an unknown proton acceptor. The proton acceptor could not be identified, but its abundance was suggested to be greatest "around the center of the spray cone" [21]. We believe that the unknown proton acceptor is likely a mixture of solvent species from the electrosprayed solution.

With thermospray ionization (TSP), it is well established that the observed mass spectrum contains species that are products of gas-phase ion-molecule reactions [22, 23]. It is also known that multiply charged ions, both positively and negatively charged, can be observed in TSP mass spectra [24, 25]. Recently, Straub and Chan [26] have reported the observation of multiply protonated proteins, for example, myoglobin and cytochrome $c$, using TSP. Compared to the ESI mass spectra for these species, there is considerably less charging observed with TSP. While the degree of charging during the primary ionization step in TSP is almost certainly an important factor [27] upon the observed charge state distribution, the TSP conditions involve considerably higher temperatures and far greater partial pressures of sovlent than ESI, and might be expected to facilitate proton transfer reactions. Therefore, the observed charge state distribution of a multiply charged species produced via TSP may be, at least in part, affected by the reaction environment (i.e., nature and concentration of solvent species) prior to analysis.

\section{Conclusions}

Gas-phase proton transfer reactions between multiply charged protein ions and $\mathrm{H}_{2} \mathrm{O}$, which would be expected to be highly endothermic on the basis of proton affinity values for the individual amino acid residues, proceed due to Coulombic destabilization phenomena. The rates of these reactions have not been established, although they are obviously much smaller than collision-limited proton transfer reactions. The reactivity of the various amino acid residues in a gas-phase multiply protonated protein molecule differ from the independent amino acids because of the contribution of Coulombic repulsion. Each proton transfer reaction serves to lower the reactivity (or increase the short range proton affinity) of the remaining protonated sites. The barrier lowering towards proton transfer contributes to the ease at which the aforementioned reactions proceed. Recent ab initio calculations of electric field effects on proton transfer reactions are qualitatively consistent [28]. Also, the elimination of a charge site, that is, a proton, can result in the reduction of the Coulombic repulsion, therefore increasing the range of allowed conformations for the ion. Coupled with the reaction barrier-lowering mechanism, this increase in entropy can apparently be sufficiently large to promote the proton transfer process.

The results presented here imply that the design of an ESI/mass spectrometry interface (and, in general, high-pressure sources producing multiply charged molecules) can markedly affect the observed charge state distribution. An interface that can efficiently diminish the amount of ion solvation, as well as limit the amount of solvent entering the mass spectrometer, will be capable of producing a mass spectrum of a multiply protonated species that more closely resembles the ion population initially formed. This, in turn, affects the resulting signal intensities and necessarily the massto-charge ratio range, and may affect the ability to observe small changes in the charge state distribution arising from differences in the net charge in solution.

\section{Acknowledgments}

We thank Dr. A. L. Rockwood for helpful discussions regarding the concept of "apparent proton affinities" and the U.S. Department of Energy, Office of Health and Environmental Research and the Molecular Sciences Research Center for support of this research under contract DE-AC06-76RLO 1830. Pacific Northwest Laboratory is operated by Battelle Memorial Institute.

\section{References}

1. Dole, M.; Mack, L. L.; Hines, R. L.; Mobley, R. C.; Ferguson, L. D.; Alice, M. B. Chem. Phys. 1968, 3, 2240.

2. Yamishita, M.; Fenn, J. B. J. Phys. Chem. 1984, 88, 4451. Fenn, J. B.; Mann, M.; Meng, C. K.; Wong, S. F.; Whitehouse, C. M. Science 1989, 246, 64. Fenn, J. B.; Mann, M.; Meng, C. K.; Wong, S. F. Mass Spectrom. Rev. 1990, 9, 37. 
3. Loo, J. A.; Ldseth, H. R.; Smith, R. D. Biomed. Environ. Mass Spectrom. 1988, 17, 411. Smith, R. D.; Loo, J. A.; Edmonds, C. G.; Barinaga, C. J.; Udseth, H. R. Attal. Chem. 1990, 62, 882.

4. Bruins. A. P,; Covey, T. R.; Henion, J. D. Anal. Chem. 1987, $98,2642$.

5. Nohmi, T,; Fenn, J. B. J. Amer. Chem. Soc, 1992, 114, 3241.

6. McLuckey, S. A.; Van Berkel, G. J.; Glish, G. L. I. Am. Chem. Soc. 1990, 112, 5668.

7. McLuckey, S. A.; Glish, G. L.; Van Berkel, G. J. Anal. Chem. 1991, 63, 1971.

8. Locke, M.; Mclver, R. T. Jr. J. Am. Chem. Sac. 1983, 105, 4226.

9. Ogorzalek Loo, R. R.; Udseth, H. R.; Smith, R. D. J. Phys. Chem. 1991, 95, 6412.

10. Ogorzalek Loo, R. R.; Loo, J. A.; Udseth, H. R.; Smith, R. D. Rapid Commun. Mass Spectrom, 1992, 6, 159.

11. Chait, B. T.; Chowdhury, S. K.; Katta, V. In Proceedings of the 39th Conference on Mass Spectrometry and Allied Topics, Nashville, TN, 1991; p 447.

12. Smith, R. D.; Olivares, J. A.; Nguyen, N. T.; Udseth, H. R. Anal. Chem. 1988, 60, 436. Loo, J. A.; Udseth, H. R.; Smith, R. D. Anal. Biochem. 1989, 179, 404.

13. Smith, R. D.; Loo, J. A.; Barinaga, C. J:; Edmonds, C. G.; Udseth, H. R. I. Amer. Soc. Mass Spectrom. 1990, 1, 53.

14. Vacuum Technology: Its Foundations, Formulae, and Tables; Leybold, Inc. Reference Book, 1990; p 51.
15. Roche, A. E.; Sutton, M. M.; Bohne, D. K.; Schiff, H. I. J. Chem. Phys. 1971, 55, 5480 .

16. Wolf, J. F.; Staley, R. H.; Koppel, I.; Taaqepera, M.; McIver, R. T. Jr.; Beauchamp, J. L.; Raft, R. W. J. Am. Chem. Soc. 1977 99, 5417.

17. Meot-Ner, M. J. Phys. Chem. 1991, 95, 6580.

18. Meot-Ner, M.; Hunter, E.; Field, F. I. Am. Chem. Soc. 1979, $101,686$.

19. Gorman, G. S.; Speir, J. P.; Turner, C. A.; Amster, I. J. J. Amer. Chem. Soc, 1992 114, 3986.

20. Rockwood, A. L.; Busman, M.; Smith, R. D. Int. J. Mass Spectrom. Ion. Proc. 1991, 111, 103.

21. Hiraoka, K.; Judaka, I. Anal Chem. 1992, 64, 75.

22. Bursey, M. M.; Parker, C. E.; Smith, R. W.; Gaskell, S. J. Anal. Chem. 1985, 57, 2597.

23. Alexander, A. J.; Kebarle, P. Anal. Chem. 1986, 58, 471.

24. Blakely, C. R.; Vestal, M. L. Anal. Chem. 1983, 55, 750.

25. Thomson, B. A.; Iribarne, J. V.; Dziedzic, P. J. Anal. Chem. 1982, 54,2219

26. Straub, K.; Chan, K. Rapid Commun. Mass Spectrom. 1990, 4, 267.

27. Katta, V.; Rockwood, A. L,: Vestal, M. L. Int. J. Mass Spectrom. Ion Proc. 1991, 103, 129

28. Rockwood, A. L.; Smith, R. D. Presented at the 40th ASMS Conference on Mass Spectrometry and Allied Topics, Washington, DC, May 31-June 5, 1992. 